International Journal of Modern Physics B,

(C) World Scientific Publishing Company

\title{
BETHE ANSATZ AND CLASSICAL HIROTA EQUATION
}

\author{
P. WIEGMANN \\ James Franck Institute and Enrico Fermi Institute \\ University of Chicago, Chicago, IL 60637, USA
}

\begin{abstract}
We discuss an interrelation between quantum integrable models and classical soliton equations with discretized time. It appeared that spectral characteristics of quantum integrable systems may be obtained from entirely classical set up. Namely, the eigenvalues of the quantum transfer matrix and the scattering $S$-matrix itself are identified with a certain $\tau$-functions of the discrete Liouville equation. The Bethe ansatz equations are obtained as dynamics of zeros. For comparison we also present the Bethe ansatz equations for elliptic solutions of the classical discrete Sine-Gordon equation. The paper is baped on the recent study of classical integrable structures in quantum integrable systems.
\end{abstract}

\section{Introduction}

1. In 1981 Hirotal proposed a difference equation which unifies all known continuous soliton equations. A particular case of the Hirota equation is a bilinear difference equation for a function $\tau(n, l, m)$ of three discrete variables:

$$
\begin{aligned}
\alpha \tau(n, l+1, m) \tau(n, l, m+1)+\beta \tau(n, l, m) \tau(n, l+1, m+1) & \\
& +\gamma \tau(n+1, l+1, m) \tau(n-1, l, m+1)=0,
\end{aligned}
$$

where it is assumed that $\alpha+\beta+\gamma=0$. Different continuum limits at different boundary conditions then reproduce continuous soliton equations (Kadomtsev-Petviashvili equation, Toda lattice, etc.). On the other hand, $\tau(n, l, m)$ can be identified 3 with the $\tau$-function of a continuous hierarchy.

The same equation (with a particular boundary condition) has suddenly appeared in the theory of quantum integrable systems as a fusion relation for the transfer matrix (trace of the quantum monodromy matrix).

Thus classical integrability emerges in quantum systems not as a limiting procedure but rather as inherent and exact. It appears that while solving an integrable quantum problem we in fact deal with classical integrable equations, but with a discretized time. Indeed, the Bethe ansatz equations and the eigenvalues of the S-matrix and transfer matrix may be obtained by solving classical Hirota equation.

At present, we cannot treat an appearance of the classical (discrete time) equations in quantum problem better than an observation, although all this indicates that classical and quantum integrable systems have much more in common than a regular classical limit $\hbar \rightarrow 0$.

2. The transfer matrix is one of the key objects in the theory of quantum integrable systems 6 Transfer matrices form a commutative family of operators acting 
in the Hilbert space of a quantum problem. Let $R_{i, \mathcal{A}}(u)$ be the $R$-matrix acting in the tensor product of Hilbert spaces $V_{i} \otimes V_{\mathcal{A}}$. Then the transfer matrix is a trace over the auxiliary space $V_{\mathcal{A}}$ of the monodromy matrix that is the matrix product of $N R$-matrices with the common auxiliary space:

$$
\begin{aligned}
\hat{T}_{\mathcal{A}}\left(u \mid y_{i}\right) & =R_{N, \mathcal{A}}\left(u-y_{N}\right) \cdots R_{2, \mathcal{A}}\left(u-y_{2}\right) R_{1, \mathcal{A}}\left(u-y_{1}\right), \\
T_{\mathcal{A}}(u) & =\operatorname{tr}_{\mathcal{A}} \hat{T}_{\mathcal{A}}\left(u \mid y_{i}\right) .
\end{aligned}
$$

The transfer matrices commute for all values of the spectral parameter $u$ and different auxiliary spaces:

$$
\left[T_{\mathcal{A}}(u), T_{\mathcal{A}^{\prime}}\left(u^{\prime}\right)\right]=0 .
$$

They can be diagonalized simultaneously, i.e. their eigenstates do not depend on $\mathcal{A}$ and $u$. The family of eigenvalues of the transfer matrix is the first question to be addressed for an integrable system - the spectrum of a quantum problem can be expressed in terms of eigenvalues of the transfer matrix.

Moreover, the transfer matrix may be used to obtain the scattering $S$-matrix. Indeed, at $u=0$ the fundamental $R$-matrix becomes a permutation $R(u=0)=P$. Therefore, choosing $\mathcal{A}$ to be the same as the representation of , say, the first particle and setting $u=y_{1}$, the transfer matrix becomes the $S$-matrix of scattering the first particle with rapidity $y_{1}$ by the other $N-1$ particles

$$
S_{\mathcal{A}}\left(u \mid y_{2}, \ldots y_{N}\right)=R_{N, \mathcal{A}}\left(u-y_{N}\right) \ldots R_{2, \mathcal{A}}\left(u-y_{2}\right) .
$$

3. The transfer matrix corresponding to a given representation in the auxiliary space can be constructed out of transfer matrices for some elementary space by means of the fusion procedure. at certain values of the spectral parameter $u$ the $R$-matrix becomes essentially a projector onto an irreducible representation space. The fusion rules are especially simple in the $A_{1}$-case. Consider for example the rational solution of the Yang-Baxter equation for the fundamental (spinor) representations of $S U(2)$ :

$$
R_{1,1}(u)=u-2 P
$$

where $P$ is the permutation operator. At a special value of the spectral parameter $u= \pm 2$, the $R$-matrix becomes a projector onto irreducible moduli of the tensor product $[1 / 2] \otimes[1 / 2]=[0] \oplus[1]$ - the singlet (spin-0) at and onto the triplet (spin-1) $: R( \pm 2)=4 P^{\mp}$. Then the transfer matrix $T_{2}^{1}(u)$ with spin-1 auxiliary space is obtained from the product of two spin- $1 / 2$ monodromy matrices $\hat{T}_{1}^{1}(u)$ with arguments shifted by 2 :

$$
T_{2}^{1}(u)=\operatorname{tr}_{[1]}\left(R_{1,1}(-2) \hat{T}_{1}^{1}(u+1) \hat{T}_{1}^{1}(u-1) R_{1,1}(-2)\right) .
$$

A combination of the fusion procedure and the Yang-Baxter equation results in numerous functional relations (fusion rules) for the transfer matrix 7 They were recently combined into a universal bilinear form. 
have the most simple closed form for the models of the $A_{k-1}$-series and representations corresponding to rectangular Young diagrams.

Let $T_{s}^{a}(u)$ be the transfer matrix for the rectangular Young diagram of length $a$ and height $s$. They obey the following bilinear functional relation:

$$
T_{s}^{a}(u+1) T_{s}^{a}(u-1)-T_{s+1}^{a}(u) T_{s-1}^{a}(u)=T_{s}^{a+1}(u) T_{s}^{a-1}(u) .
$$

Since $T_{s}^{a}(u)$ commute at different $u, a, s$, the same equation holds for eigenvalues of the transfer matrices, so we can (and will) treat $T_{s}^{a}(u)$ in (6) as number-valued functions. The bilinear fusion relations for models related to other Dynkin graphs were suggested in ref. $\mathrm{D}$.

Remarkably, the bilinear fusion relations (6) appear to be identical to the Hirota equation (11). Indeed, one can eliminate the constants $\alpha, \beta, \gamma$ by the transformation

$$
\tau(n, l, m)=\frac{(-\alpha / \gamma)^{n^{2} / 2}}{(1+\gamma / \alpha)^{l m}} \tau_{n}(l, m)
$$

so that

$$
\begin{aligned}
\tau_{n}(l+1, m) \tau_{n}(l, m+1)-\tau_{n}(l, m) \tau_{n}(l+1, m+1) & \\
& -\tau_{n+1}(l+1, m) \tau_{n-1}(l, m+1)=0
\end{aligned}
$$

and then change variables from light-cone coordinates $n, l, m$ to the direct variables

$$
\begin{array}{r}
a=n, \quad s=l+m, \quad u=l-m-n, \\
\tau_{n}(l, m) \equiv T_{l+m}^{a}(l-m-n) .
\end{array}
$$

At least at a formal level, this transformation provides the equivalence between (6), (11) and (7).

Relation between Hirota's equations and fusion relations goes much further. It is known that a general class of Hirota's equations are related with the geometry of Grassmannian manifolds and can be constructed out of a general Young tableau.10.11 If, for example, the Young diagrams $Y=\left[a_{1}^{s_{2}},\left(a_{1}+a_{2}\right)^{s_{1}}\right]$ consist of two rectangular blocks (one with $a_{1}$ lines of length $s_{1}+s_{2}$ and the second with $a_{2}$ lines of length $s_{1}$ ), then the higher Hirota equations hold

$$
\begin{aligned}
& T_{s_{1}, s_{2}}^{a_{1}, a_{2}-1}(u) T_{s_{1}-1, s_{2}-1}^{a_{1}, a_{2}+1}(u)+T_{s_{1}, s_{2}+1}^{a_{1}-1, a_{2}-1}(u) T_{s_{1}, s_{2}-1}^{a_{1}+1, a_{2}+1}(u) \\
+ & T_{s_{1}+1, s_{2}}^{a_{1}-1, a_{2}}(u-1) T_{s_{1}-1, s_{2}}^{a_{1}+1, a_{2}}(u+1) \\
= & T_{s_{1}+1, s_{2}}^{a_{1}, a_{2}-1}(u-1) T_{s_{1}-1, s_{2}}^{a_{1}, a_{2}+1}(u+1)+T_{s_{1}, s_{2}+1}^{a_{1}-1, a_{2}}(u-1) T_{s_{1}, s_{2}-1}^{a_{1}+1, a_{2}}(u+1) .
\end{aligned}
$$

This is also a fusion relation for the transfer matrix of the auxiliary space of the representation $Y$.

Leaving aside more fundamental aspects of this "coincidence", we exploit, as a first step, some technical advantages it offers. Specifically, we treat the functional relation (6) not as an identity but as a fundamental equation which (together with 
particular boundary and analytical conditions) completely determines all the eigenvalues of the transfer matrix. The solution of the Hirota equation then appears in the form of the Bethe ansatz equations. We anticipate that this approach makes it possible to use some specific tools of classical integrability and, in particular, the finite gap integration technique.

The origin of $T_{s}^{a}(u)$ as an eigenvalue of the transfer matrix (2) imposes specific boundary conditions:

$$
T_{s}^{a}=0, \quad a \neq 0,1, . ., k
$$

and, what is equally important, certain analytical properties. As a general consequence of the Yang-Baxter equation, the transfer matrices may be always normalized to be elliptic polynomials in the spectral parameter, i.e. finite products of Weierstrass $\sigma$-functions (as in (19) below). Solutions with that kind of analytical properties (called elliptic solutions) are well-known in the theory of classical soliton equations due to the works. $12,13.4210$

4. To illustrate elliptic solutions in classical continuum time soliton equations let us recall the result of the Ref.14 for the Kadomtsev-Petviashvili (KP) equation. A particular elliptic solution can be written in the form

$$
u(x, y, t)=2 \sum_{i=1}^{N} \mathcal{P}\left(x-x_{i}(y, t)\right)
$$

where $\mathcal{P}$ is Weierstrass elliptic function and all dynamics in $t$ and $y$ is hidden in the behaviour of poles $x_{i}(y, t)$. A very existence of this ansatz is an implementation of the integrability. It appears that poles obey the elliptic Calogero-Moser many-body system with respect to $y$

$$
x_{i}^{\prime \prime}=-\sum_{j} \mathcal{P}^{\prime}\left(x_{i}-x_{j}\right) .
$$

Elliptic solutions can be lifted up to the discrete case.1.6. Then poles $x_{i}$ (or roots of the elliptic polynomial) obey a fully discretized version of the Calogero-Moser model, which can be recognized as the Bethe ansatz equations.

In this paper we shall consider the discrete version of the classical Liouville equation. Its elliptic solutions gives the eigenvalues of the transfer matrix and the $S$-matrix of the quantum integrable systems associated with $A_{1}$ algebra. For extension to $A_{k-1}$ algebra see Ref.t. We also compare it, but do not study in detail, with Bethe ansatz solution of the discrete Sine Gordon equation. 17

The Hirota equation for the $A_{1}$ case may be obtained from the more general (6), by setting the boundary conditions

$$
T_{s}^{a}=0, \quad a \neq 0,1,2
$$

Then $T^{0,2}$ obey the discrete Laplace equation while the $T_{s}^{1}(u) \equiv T_{s}(u)$ obeys the discrete Liouville equation (we continue to refer it as the Hirota equation):

$$
T_{s}(u+1) T_{s}(u-1)-T_{s+1}(u) T_{s-1}(u)=T_{s}^{0}(u) T_{s}^{2}(u) .
$$


In fact the values of $T_{s}^{0}(u)$ and $T_{s}^{2}(u)$ can be easily determined. They are the transfer matrices in most symmetric and antisymmetric states of the $A_{2}$ case. These states have dimension 1 . In the most symmetrical state there is no scattering, so $T_{s}^{0}(u)=1$. The value of the transfer matrix in the most antisymmetric state is known as the quantum determinant 18

$$
T_{s}^{2}(u)=\frac{\gamma(u+s) \gamma(u+s+4)}{\gamma(u-s) \gamma(u-s+4)} .
$$

where

$$
\frac{\gamma(u+2)}{\gamma(u)}=\phi(u)
$$

and

$$
\phi(u)=\prod_{k=1}^{N} \prod_{i=-q_{k}+1}^{q_{k}-1} \sigma\left(\eta\left(u-y_{k}+i\right)\right) .
$$

Here $\sigma(x)$ is the Weierstrass $\sigma$-function and $q_{k}$ is a spin of the $k$ th quantum space. The roots $y_{k}$ and degree $N$ of the elliptic polynomial $\phi(u)$ and parameters of the Weierstrass function are parameters of the quantum model. Below we concentrate mostly on the simplest case $q_{k}=1$.

Apparently, different solutions of (14) have a number common zeros and $T_{s}(u)$ at general parameters $y_{k}$ are polynomials of degree $s \sum q_{k}$. These zeros can be gauged out, so all $T_{s}(u)$ (different eigenvalues at different $s$ and $q_{k}$ ) remain polynomials of the same degree $N$. The maximal gauge transformation is

$$
T_{s}(u) \rightarrow T_{s}(u) \prod_{p=2}^{s} \phi(u+s-2 p) \prod_{k=1}^{N} \prod_{p=1}^{q_{k}-s} \sigma\left(\eta\left(u-s+q_{k}+2-2 p\right)\right.
$$

where the last product holds for $q_{k}>s$. This gauge transformation simplifies the r.h.s. of (14)

$$
T_{s}(u+1) T_{s}(u-1)-T_{s+1}(u) T_{s-1}(u)=\phi(u+s) \phi(u-s-2) .
$$

In this normalization all $T_{s}$ are polynomials of the same degree with no common zeros.

A general solution of the discrete Liouville equation (14) is parametrized by two arbitrary functions of one variable. Not all of them correspond to eigenvalues of the quantum transfer matrix, but only elliptic solution. Elliptic polynomials may be characterized by its roots $z_{j}^{(s)}$

$$
T_{s}(u)=A_{s} e^{\mu_{s} u} \prod_{j=1}^{N} \sigma\left(\eta\left(u-z_{j}^{(s)}\right)\right),
$$

where $A_{s}, \mu_{s}$ do not depend on $u$. Similar to the continuum case, roots obey some dynamics in a discrete "time" variable $s$. It is determined by the Bethe-ansatz-like 
P. Wiegmann

equations

$$
\begin{aligned}
& \frac{\phi\left(z_{j}^{(s)}+s+1\right)}{\phi\left(z_{j}^{(s)}+s+3\right)}=-\frac{A_{s-1}}{A_{s+1}} \prod_{k=1}^{N} \frac{\sigma\left(\eta\left(z_{j}^{(s)}-z_{k}^{(s-1)}-1\right)\right)}{\sigma\left(\eta\left(z_{j}^{(s)}-z_{k}^{(s+1)}+1\right)\right)}, \\
& \frac{\phi\left(z_{j}^{(s)}-s+1\right)}{\phi\left(z_{j}^{(s)}-s-1\right)}=-\frac{A_{s-1}}{A_{s+1}} \prod_{k=1}^{N} \frac{\sigma\left(\eta\left(z_{j}^{(s)}-z_{k}^{(s-1)}+1\right)\right)}{\sigma\left(\eta\left(z_{j}^{(s)}-z_{k}^{(s+1)}-1\right)\right)} .
\end{aligned}
$$

The constant $A_{s}$ can be easily found in the rational and trigonometric case by comparing the leading powers of polynomials in the (18). In the rational case it obeys the equation

$$
A_{s}^{2}-A_{s+1} A_{s-1}=0 \text {. }
$$

Each solution of these equations gives an eigenvalue of the transfer matrix. Equations (20) and (21) determine the zeros of the transfer matrix. The traditional Bethe ansatz equations

$$
e^{-4 \eta \nu} \frac{\phi\left(u_{j}\right)}{\phi\left(u_{j}-2\right)}=-\prod_{k} \frac{\sigma\left(\eta\left(u_{j}-u_{k}+2\right)\right)}{\sigma\left(\eta\left(u_{j}-u_{k}-2\right)\right)} .
$$

corresponding to zeros of the Baxter $Q$ matrix are discussed in Sec. 5 .

\section{Other Forms of the Hirota Equation}

The equation (14) is known as a discrete version of the Liouville equation 19 written in terms of the $\tau$-function. It can be recast to a more universal form in terms of the discrete Liouville field

$$
Y_{s}^{1}(u) \equiv Y_{s}(u)=\frac{T_{s+1}(u) T_{s-1}(u)}{\phi(u+s) \phi(u-s-2)}
$$

which hides the function $\phi(u)$ in the r.h.s. of $(18)$. The equation becomes

$$
Y_{s}(u-1) Y_{s}(u+1)=\left(Y_{s+1}(u)+1\right)\left(Y_{s-1}(u)+1\right) .
$$

with a boundary condition $Y_{0}(u)=0$. (Let us note that the same functional equation but with differentanalytic properties of the solutions appears in the thermodynamic Bethe ansatz.29)

The functional equations (24) can be further rewritten in an integral form. Introducing $\varepsilon_{s}(u)=\ln Y_{s}(u)$, one gets

$$
\varepsilon_{s}(u)-\int_{-i \infty}^{i \infty} \frac{1}{2 \pi \cos \frac{\pi}{2}\left(u-u^{\prime}\right)} \log \left(\left(1+e_{s+1}^{\varepsilon}\right)\left(1+e_{s-1}^{\varepsilon}\right)\right)=\sum_{k} \delta_{s q_{k}} \bar{\varphi}_{k}(u)
$$

where $\bar{\varphi}_{k}(u)$ are some known functions computed out of the r.h.s. of the (14), such as $\bar{\varphi}_{k}(u+1)+\bar{\varphi}_{k}(u-1)=0.4$ 


\section{Difference and Continuum $A_{1}$-Equation}

Liouville equation. In the continuum limit one should put $Y_{s}(u)=\delta^{-2} e^{-\varphi(x, t)}$, $u=\delta^{-1} x, s=\delta^{-1} t$. An expansion in $\delta \rightarrow 0$ then gives the continuous Liouville equation

$$
\partial_{s}^{2} \varphi-\partial_{u}^{2} \varphi=2 \exp (\varphi)
$$

Sine-Gordon equation. For illustrative purposes let us compare it with discrete Sine-Gordon (SG) equation, which requires quasi-periodic boundary condition with respect to the Dynkin diagram 21

$$
T_{s}^{a+1}(u)=e^{\alpha} \lambda^{2 a} T_{s}^{a-1}(u-2),
$$

where $\alpha$ and $\lambda$ are parameters. Plugging this condition into (6), we get:

$$
\begin{gathered}
T_{s}^{1}(u+1) T_{s}^{1}(u-1)-T_{s+1}^{1}(u) T_{s-1}^{1}(u)=e^{\alpha} \lambda^{2} T_{s}^{0}(u) T_{s}^{0}(u-2), \\
T_{s}^{0}(u+1) T_{s}^{0}(u-1)-T_{s+1}^{0}(u) T_{s-1}^{0}(u)=e^{-\alpha} T_{s}^{1}(u) T_{s}^{1}(u+2) .
\end{gathered}
$$

Let us introduce two fields $\rho^{s, u}$ and $\varphi^{s, u}$ on the square $(s, u)$ lattice

$$
\begin{aligned}
T_{s}^{0}(u) & =\exp \left(\rho^{s, u}+\varphi^{s, u}\right), \\
T_{s}^{1}(u+1) & =\lambda^{1 / 2} \exp \left(\rho^{s, u}-\varphi^{s, u}\right),
\end{aligned}
$$

and substitute them into (28) and (29). Finally, eliminating $\rho^{s, u}$, one gets the discrete SG equation:

$$
\begin{aligned}
\sinh \left(\varphi^{s+1, u}\right. & \left.+\varphi^{s-1, u}-\varphi^{s, u+1}-\varphi^{s, u-1}\right) \\
& =\lambda \sinh \left(\varphi^{s+1, u}+\varphi^{s-1, u}+\varphi^{s, u+1}+\varphi^{s, u-1}+\alpha\right) .
\end{aligned}
$$

The constant $\alpha$ can be removed by the redefinition $\varphi^{s, u} \rightarrow \varphi^{s, u}-\alpha / 4$.

Another useful form of the discrete SG equation appears in variables

$$
X_{s}^{a}(u)=-\frac{T_{s}^{a}(u+1) T_{s}^{a}(u-1)}{T_{s}^{a+1}(u) T_{s}^{a-1}(u)}=-1-Y_{s}^{a}(u)
$$

Under condition (27) one has

$$
X_{s}^{a+1}(u)=X_{s}^{a-1}(u-2), \quad \lambda^{2} X_{s}^{a+1}(u+1) X_{s}^{a}(u)=1,
$$

so there is only one independent function

$$
X_{s}^{1}(u) \equiv x_{s}(u)=-e^{-\alpha} \lambda^{-1} \exp \left(-2 \varphi^{s, u}-2 \varphi^{s, u-2}\right) .
$$

The discrete SG equation becomes2, 22,23

$$
x_{s+1}(u) x_{s-1}(u)=\frac{\left(\lambda+x_{s}(u+1)\right)\left(\lambda+x_{s}(u-1)\right)}{\left(1+\lambda x_{s}(u+1)\right)\left(1+\lambda x_{s}(u-1)\right)} .
$$


P. Wiegmann

In the limit $\lambda \rightarrow 0$ (36) turns into the discrete Liouville equation (24) for $Y_{s}(u)=$ $-1-\lambda^{-1} x_{s}(u)$.

\section{Determinant representations}

Relation between the Hirota equation and Plucker relations of the coordinates of the Grassmannian manifolds suggests a numerous determinant representations of its solutions. The most familiar one 24 allows one to express $T_{s}^{a}(u)$ through $T_{1}^{a}(u)$ or $T_{s}^{1}(u)$. For instance the determinant formula giving the evolution in $a$ holds

$$
T_{s}^{a}(u)=\operatorname{det}\left(T_{1}^{a+i-j}(u+i+j-s-1)\right), \quad i, j=1, \ldots, s, \quad T_{0}^{a}(u)=1 .
$$

Another representation appeared to be more suitable for particularities of the $A_{k-1}$ Liouville equation (6) and (10). They explicitly express its solution in terms of $2 k$ arbitrary functions of one variable 1 as a determinant of the $k \times k$ matrix

$$
\begin{aligned}
\tau_{a}(l, m) & =\operatorname{det} M_{i j}, \\
M_{j i} & = \begin{cases}h_{i}(u+s+a+2 j) & \text { if } j=1, \ldots, k-a ; i=1, \ldots, k \\
\bar{h}_{i}(u-s+a+2 j) & \text { if } j=k-a+1, \ldots, k ; i=1, \ldots, k\end{cases}
\end{aligned}
$$

In the $A_{1}$ case the determinant has a particular simple form:

$$
\begin{gathered}
T_{s}^{0}(u) \equiv \phi(u+s)=\left|\begin{array}{ll}
R(u+s) & Q(u+s) \\
R(u+2+s) & Q(u+2+s)
\end{array}\right|, \\
T_{s}^{2}(u) \equiv \phi(u-s-2)=\left|\begin{array}{ll}
\bar{R}(u-s-1) & \bar{Q}(u-s-1) \\
\bar{R}(u-s+1) & \bar{Q}(u-s+1)
\end{array}\right| . \\
T_{s}(u)=\left|\begin{array}{ll}
Q(u+s+1) & R(u+s+1) \\
\bar{Q}(u-s) & \bar{R}(u-s)
\end{array}\right| .
\end{gathered}
$$

Compare first two equations one finds

$$
\bar{Q}(u)=Q(u-1), \quad \bar{R}(u)=R(u-1),
$$

so that

$$
T_{s}(u)=\left|\begin{array}{ll}
Q(u+s+1) & R(u+s+1) \\
Q(u-s-1) & R(u-s-1)
\end{array}\right|
$$

\section{Bethe-Ansatz}

The functions $Q, R$ are further determined by the r.h.s. of the (18) and by the analyticity requirement that $T_{s}(u)$ is an elliptic polynomial (19). One can show that it also means that $Q(u)$ or $R(u)$ (but not necessarily all together) is an elliptic polynomials of degree $N / 2$ multiplied by exponential function

$$
Q(u)=e^{\nu \eta u} \prod_{j=1}^{M} \sigma\left(\eta\left(u-u_{j}\right)\right)
$$


Let $u_{j}, j=1, \ldots, M$ be zeros of $Q(u)$. Then, evaluating (39) at $u=u_{j}$, $u=u_{j}-2$, we obtain the relations

$$
\phi\left(u_{j}\right)=Q\left(u_{j}+2\right) R\left(u_{j}\right), \quad \phi\left(u_{j}-2\right)=-Q\left(u_{j}-2\right) R\left(u_{j}\right),
$$

whence it holds

$$
\frac{\phi\left(u_{j}\right)}{\phi\left(u_{j}-2\right)}=-\frac{Q\left(u_{j}+2\right)}{Q\left(u_{j}-2\right)},
$$

Equation (46) is the celebrated Bethe ansatz equation (22).

In the elliptic case the degrees of the elliptic polynomial $Q(u)$ (for even $N$ ) is equal to $M=N / 2$, provided that $\eta$ is incommensurable with the lattice spanned by $\omega_{1}, \omega_{2}$. In the trigonometric and rational cases there are no such strong restrictions on degrees $M$ and $\tilde{M}$ of $Q$. This is because a part of their zeros may tend to infinity thus reducing the degree. Whence $M$ and $\tilde{M}$ can be arbitrary integers not exceeding $N$. However, they must be complement to each other: $M+\tilde{M}=N$. The traditional choice is $M \leq N / 2$. In particular, the solution $Q(u)=1(M=0)$ corresponds to the simplest reference state ("bare vacuum") of the model.

\section{Linear Problem}

The function $Q$ naturally appears as the object of the linear problem of the discrete Liouville equation. Indeed, the equation (14) admits a zero curvature representation. Let us start from a more general equation

$$
T_{s}(u+1) T_{s}(u-1)-T_{s+1}(u) T_{s-1}(u)=\phi(u+s) \bar{\phi}(u-s),
$$

with two independent functions $\phi, \bar{\phi}$ and later impose the relation $\bar{\phi}(u)=\phi(u-2)$. The nonlinear equation appears as a consistency conditions of the following auxiliary linear problems

$$
\begin{gathered}
T_{s+1}(u) Q(u+s)-T_{s}(u-1) Q(u+s+2)=\phi(u+s) \bar{Q}(u-s-1), \\
T_{s+1}(u) \bar{Q}(u-s+1)-T_{s}(u+1) \bar{Q}(u-s-1)=\bar{\phi}(u-s) Q(u+s+2),
\end{gathered}
$$

where we introduced two functions of one variable $Q(u)$ and $\bar{Q}(u)$. These are the functions appeared in the determinant representations (39), (40), and (41).

The fact that functions of the linear problem depend on one variable is a specific feature of the Liouville equation.

To compare let us present the linear problem for the discrete Sine-Gordon equation (28) and (29). It reads

$$
\begin{gathered}
T_{s+1}^{1}(u) F_{s}^{0}(u)-T_{s}^{1}(u-1) F_{s+1}^{0}(u+1)=T_{s}^{0}(u) F_{s+1}^{1}(u), \\
T_{s+1}^{0}(u-2) F_{s}^{1}(u)-T_{s}^{0}(u-3) F_{s+1}^{1}(u+1)=T_{s}^{1}(u) F_{s+1}^{0}(u-2) \\
T_{s+1}^{0}(u) F_{s}^{0}(u)-T_{s}^{0}(u-1) F_{s+1}^{0}(u-1)=e^{-\alpha} T_{s}^{1}(u) F_{s+1}^{1}(u+2), \\
T_{s+1}^{1}(u-1) F_{s}^{1}(u)-T_{s}^{1}(u) F_{s+1}^{1}(u-1)=e^{\alpha} \lambda^{2} T_{s}^{0}(u-3) F_{s+1}^{0}(u)
\end{gathered}
$$


In this case the wave functions $F_{s}^{0}(u)$ and $F_{s}^{1}(u)$ depend on both $s$ and $u$.

\section{The Baxter Relation}

A general solution of the discrete Liouville equation (for arbitrary $\phi$ and $\bar{\phi}$ ) may be expressed through two independent functions $Q(u)$ and $\bar{Q}(u)$. Below we follow the same lines developed for solving continuous classical Liouville equation (see, e.g., Refs.25.26 and references therein). First we rearrange (48) and (49) as

$$
\begin{gathered}
\phi(u-2) Q(u+2)+\phi(u) Q(u-2)=A(u) Q(u), \\
\bar{\phi}(u) \bar{Q}(u+3)+\bar{\phi}(u+2) \bar{Q}(u-1)=\bar{A}(u) \bar{Q}(u+1),
\end{gathered}
$$

where we have introduced the quantities

$$
\begin{aligned}
& A(u)=\frac{\phi(u-2) T_{s+1}(u-s)+\phi(u) T_{s-1}(u-s-2)}{T_{s}(u-s-1)}, \\
& \bar{A}(u)=\frac{\bar{\phi}(u+2) T_{s+1}(u+s)+\bar{\phi}(u) T_{s-1}(u+s+2)}{T_{s}(u+s+1)} .
\end{aligned}
$$

Due to the consistency condition (47), $A(u)$ and $\bar{A}(u)$ are functions of one variable and do not depend on $s$. The symmetry between $u$ and $s$ allows one to construct similar objects which in their turn do not depend on $u$. Functions $A(u)$ and $\bar{A}(u)$, in the r.h.s. of (54) and (55) are the conservation laws of the $s$-dynamics.

Running ahead, let us note that the connection between $\phi$ and $\bar{\phi}, \bar{\phi}(u)=\phi(u-2)$, and its consequence $T_{-1}(u)=0$, simplifies (54) - 57). Putting $s=0$ and using the boundary condition $T_{-1}(u)=0$, we find

$$
A(u)=\bar{A}(u)=T_{1}(u) .
$$

Therefore, we have the relations

$$
\begin{gathered}
T_{s}(u-1) T_{1}(u+s)=\phi(u+s-2) T_{s+1}(u)+\phi(u+s) T_{s-1}(u-2), \\
T_{s}(u+1) T_{1}(u-s)=\phi(u-s) T_{s+1}(u)+\phi(u-s-2) T_{s-1}(u+2), \\
\phi(u-2) Q(u+2)+\phi(u) Q(u-2)=T_{1}(u) Q(u) .
\end{gathered}
$$

These equalities are also known as fusion relations and have been obtained in Refs. 27.28, and (61) is also known as the Baxter $T-Q$ relation.29 So the Baxter $Q$ function and $T-Q$ relation naturally appear in the context of the auxiliary linear problems for the bilinear fusion relation.

The Baxter equation (61) gives rise to a generalized spectral problem for a discrete operator of the second order: find all elliptic "eigenvalues" of the operator $\left.\phi(u-2) e^{2 \partial_{u}}+\phi(u)\right) e^{-2 \partial_{u}}$. One may show that in this case the eigenfunction $Q(u)$ itself is an elliptic polynomial. The Bethe ansatz is a practical tool to solve this problem. 
Let us consider (54) (resp. (55)) as a second order linear difference equation, where the function $A(u)(\bar{A}(u))$ is determined from the initial data. Let $R(u)$ (resp. $\bar{R}(u)$ ) be a second (linearly independent) solution of (54) (resp. (55)) normalized so that the wronskians are

$$
\begin{gathered}
W(u)=\left|\begin{array}{ll}
R(u) & Q(u) \\
R(u+2) & Q(u+2)
\end{array}\right|=\phi(u), \\
\bar{W}(u)=\left|\begin{array}{ll}
\bar{R}(u) & \bar{Q}(u) \\
\bar{R}(u+2) & \bar{Q}(u+2)
\end{array}\right|=\bar{\phi}(u+1) .
\end{gathered}
$$

Then the general solution of (47) is given by the determinant (41).

For any given $Q(u)$ and $\bar{Q}(u)$ the second solution $R(u)$ and $\bar{R}(u)$ (defined modulo a linear transformation $R(u) \rightarrow R(u)+\alpha Q(u)$ ) can be explicitly found out of the first order recurrence relations (39), (40), if necessary. Then (41) determines $T_{s}(u)$ only in terms of $Q(u)$ :

$$
T_{s}(u)=Q(u+s+1) Q(u-s-1) \sum_{j=0}^{s} \frac{\phi(u-s+2 j-1)}{Q(u-s+2 j+1) Q(u-s+2 j-1)} .
$$

This formula has been obtained in Refs 27,28 by direct resolving fusion recurrence relations (59) and (60). The Bethe ansatz equations may be also obtained as a conditions of cancelation of poles in (64).

Let us list some more useful representations.

The conserved quantities $A(u)$ and $\bar{A}(u)$ in terms of $Q$ and $R$ have the form:

$$
\begin{aligned}
& A(u)=Q(u+2) R(u-2)-R(u+2) Q(u-2), \\
& \bar{A}(u)=\bar{R}(u+3) \bar{Q}(u-1)-\bar{Q}(u+3) \bar{R}(u-1),
\end{aligned}
$$

which are direct corollaries of (54) and (55).

In its turn Baxter's relation (61) and its "chiral" versions (54) and (55) also enjoy some determinant representation:

$$
\begin{aligned}
& \left|\begin{array}{lll}
T_{s}(u) & T_{s+1}(u-1) & Q(u+s+1) \\
T_{s+1}(u+1) & T_{s+2}(u) & Q(u+s+3) \\
T_{s+2}(u+2) & T_{s+3}(u+1) & Q(u+s+5)
\end{array}\right|=0, \\
& \left|\begin{array}{lll}
T_{s}(u) & T_{s+1}(u+1) & \bar{Q}(u-s) \\
T_{s+1}(u-1) & T_{s+2}(u) & \bar{Q}(u-s-2) \\
T_{s+2}(u-2) & T_{s+3}(u-1) & \bar{Q}(u-s-4)
\end{array}\right|=0,
\end{aligned}
$$

provided that $T_{s}(u)$ obeys the Hirota equation. 


\section{Zeros of the transfer matrix}

The linear problem (48) and (49) are convenient to find the Bethe-ansatz-like equations (19) for zeros of $T_{s}(u)$. Substituting $u=z_{i}^{(s+1)}$ and $u=z_{i}^{(s)}+1$ into (48) and $u=z_{i}^{(s+1)}$ and $u=z_{i}^{(s)}-1$ into (49) we obtained the (20) and (21) referred in the introduction. In the rational case $(\sigma(\eta u) \rightarrow u) A_{s}=s+1$.

Let us compare the Bethe-ansatz-like equations of the Liouville equation (20) and (21) with the Sine-Gordon equation (28) and (29). Let $z_{i}^{0}(s)$ and $z_{j}^{1}(s)$ be the zeros of the elliptic polynomial

$$
T_{s}^{1,2}(u)=A_{s}^{1,2} e^{\mu(s) u} \prod_{j=1}^{N} \sigma\left(\eta\left(u-z_{j}^{1,2}(s)\right)\right) .
$$

Substituting $u=z_{i}^{1,2}(s)$ into the second pair of equations of the linear problem (52) and (53) and excluding $F$ 's after some algebra one obtains

$$
\begin{gathered}
\prod_{j=1}^{N}\left(\frac{\sigma\left(\eta\left(z_{i}^{1}(s+1)-z_{j}^{0}(s+1)-1\right)\right.}{\sigma\left(\eta\left(z_{i}^{1}(s+1)-z_{j}^{0}(s+2)-3\right)\right.}\right)\left(\frac{\sigma\left(\eta\left(z_{i}^{1}(s)-z_{j}^{1}(s)+1\right)\right.}{\sigma\left(\eta\left(z_{i}^{1}(s+1)-z_{j}^{1}(s+1)+1\right)\right.}\right) \\
\times \frac{\sigma\left(\eta\left(z_{i}^{1}(s+1)-z_{j}^{0}(s+2)-2\right)\right.}{\sigma\left(\eta\left(z_{i}^{1}(s+1)-z_{j}^{0}(s)\right)\right.}=-\frac{A_{s}^{0} A_{s+1}^{1}}{A_{s+1}^{0} A_{s}^{1}} \\
\prod_{j=1}^{N}\left(\frac{\sigma\left(\eta\left(z_{i}^{0}(s+1)-z_{j}^{1}(s+1)+2\right)\right.}{\sigma\left(\eta\left(z_{i}^{0}(s+1)-z_{j}^{1}(s+1)+1\right)\right.}\right)\left(\frac{\sigma\left(\eta\left(z_{i}^{0}(s)-z_{j}^{0}(s+1)+1\right)\right.}{\sigma\left(\eta\left(z_{i}^{0}(s)-z_{j}^{0}(s)+1\right)\right.}\right) \\
\times \frac{\sigma\left(\eta\left(z_{i}^{0}(s+1)-z_{j}^{1}(s+2)-1\right)\right.}{\sigma\left(\eta\left(z_{i}^{0}(s+2)-z_{j}^{1}(s+2)\right)\right.}=-\frac{A_{s}^{0} A_{s+1}^{1}}{A_{s+1}^{0} A_{s}^{1}}
\end{gathered}
$$

The constants $A_{s}$ may be easily found in the rational or trigonometric limits by comparing leading orders of polynomials in the Hirota equation. In the rational case one gets:

$$
\begin{gathered}
\left(A_{s}^{1}\right)^{2}-A_{s+1}^{1} A_{s-1}^{1}=e^{\alpha} \lambda^{2}\left(A_{s}^{0}\right)^{2} \\
\left(A_{s}^{0}\right)^{2}-A_{s+1}^{0} A_{s-1}^{0}=e^{-\alpha} \lambda^{2}\left(A_{s}^{1}\right)^{2} .
\end{gathered}
$$

These equations gives a discrete dynamics of zeros in $s$. They are to be compared with dynamics of zeros of elliptic solutions of classical nonlinear equations. 22 . 23.14 .15

\section{Crossing Symmetry and the S-Matrix}

As it was noticed in the introduction, once the eigenvalues of the transfer matrix are found it is easy to get eigenvalues of the $S$-matrix (4). In relativistic field theories the $S$-matrix is normalized in a way to be unitary and crossinvariant, i.e. to be unitary in both $s$ and $t$ channels.

Let us consider for instance the spin $1 / 2$ fundamental $A_{1}$ rational $S$-matrix. It has the form:

$$
S(u)=U(u) R(u)
$$


where the $R$-matrix is given by (5) and the amplitude $U(u)$ is restricted by the unitarity and the cross symmetry. The unitarity conditions in both channels ard 30

$$
\begin{gathered}
U(u) U^{*}(-u)=\frac{1}{\phi(u+2) \phi(-u+2)} \\
\frac{U(u+2)}{U(u-2)}=\frac{\phi(u+2) \phi(u-2)}{\phi(u) \phi(u+4)} .
\end{gathered}
$$

The minimal solution is

$$
U(u)=\frac{\Gamma\left(\frac{u+4}{4}\right) \Gamma\left(\frac{2-u}{4}\right)}{(u+2) \Gamma\left(\frac{4-u}{4}\right) \Gamma\left(\frac{u+2}{4}\right)} .
$$

The Hirota equation (18) has a natural normalization which gives the crossinvariant unitary $S$-matrices in all representations. It is a gauge which brings the quantum determinant (15) to 1 and the Hirota equation and the Baxter relation (61) to the universal form

$$
\begin{gathered}
\tau_{s}(u+1) \tau_{s}(u-1)-\tau_{s+1}(u) \tau_{s-1}(u)=1 \\
\tilde{Q}(u+2)+\tilde{Q}(u-2)=\tau_{1}(u) \tilde{Q}(u) .
\end{gathered}
$$

This is achieved by the gauge

$$
T_{s}(u)=\tau_{s}(u) \frac{f(u+s)}{f(u-s)}
$$

where

$$
f(u+1) f(u-1)=\gamma(u) \gamma(u+4) .
$$

Then $\tau_{s}$ is a meromorphic function with prescribed positions of poles. It brings the $R$-matrix to the cross unitary $S$-matrix

$$
S(u)=\frac{f(u+1)}{f(u-1)} R(u) .
$$

This factor can be identified with the factor $U$ in (25). Indeed, (81) implies

$$
\begin{gathered}
\frac{f(u+3)}{f(u-1)}=\phi(u) \phi(u+4) \\
\frac{f(u+3) f(u-3)}{f(u+1) f(u-1)}=\frac{\phi(u) \phi(u+4)}{\phi(u-2) \phi(u+2)}
\end{gathered}
$$

This is equivalent to the unitarity and cross-unitarity relation (75) and (76).

\section{Conclusion and Outlook}

It turned out that quantum integrable models have a deep connection with classical discrete soliton equations: the fusion rules for quantum transfer matrices are identical to the Hirota bilinear difference equation with a certain boundary conditions 
and analytical requirement. This coincidence goes far beyond the simplest example considered in this paper. It most likely holds for all Lie algebras and representations and all integrable boundary conditions.

Under this identification eigenvalues of the transfer matrix are represented as $\tau$-functions. Positions of zeroes of the solution is determined by the Bethe ansatz equations. We therefore conclude that the information one usually obtains from the Yang-Baxter equation may be also found from classical discrete time equations, without implying a quantization.

In general the Bethe ansatz technique, what has been thought of as a specific tool of quantum integrability is shown to exist in classical discrete nonlinear integrable equations. We demonstrated this on two examples - discrete Liouville and discrete Sine-Gordon equation. It is tempting to compare solutions of the quantum SineGordon field theory and discrete classical Sine-Gordon equation. In a forthcoming paper we extend the theory of elliptic solution known in continuum soliton equations to a more general class of discrete equations by means of the Bethe ansatz.

The difference bilinear equation, although with different analytical requirements, has appeared in quantum integrable systems in another context. Spin-spin correlation functions of the Ising model obey a bilinear difference equation that can be recast into the form of Hirota equation. 31.32 .33 More recently, nonlinear equations for correlation functions have been derived for more general class of quantum integrable models, by virtue a new approach of the Ref 34 . Thermodynamic Bethe ansatz equations written in a form of functional relations 20 (see, e.g., Ref.35) appeared to be identical with Hirota equation (although with another analytic properties) as well.

All these suggest that the Hirota equation may play a role of a master equation simultaneously for both classical and quantum integrable systems, such as the "equivalence" between quantum systems and discrete classical dynamics may be extended beyond the spectral properties discussed in this paper.

\section{Acknowledgements}

This work has been supported in part by NSF grant DMR-9509533. The author thanks A. Luther for his hospitality in NORDITA in August 1996 where this paper has been written.

\section{References}

1. I. Krichever, O. Lipan, P. Wiegmann and A. Zabrodin, "Quantum Integrable Systems and Elliptic Solutions of Classical Discrete Nonlinear Equations", preprint hepth/9604080

2. R. Hirota, J. Phys. Soc. Japan 50, 3785 (1981).

3. T. Miwa, Proc. Japan Acad. 58, 9 (1982).

4. A. Klümper and P. Pearce, Physica A183, 304 (1992).

5. A. Kuniba, T. Nakanishi and J. Suzuki, Int. J. Mod. Phys. A9 5215 (1994).

6. L. D. Faddeev and L. A. Takhtadjan, Uspekhi Mat. Nauk 34, 13 (1979).

7. P. P. Kulish and N. Yu. Reshetikhin, Zap. Nauchn. Sem. LOMI 120, 92 (1982) (in Russian). 
8. P. P. Kulish and E. K. Sklyanin, Lecture Notes in Physics 151, 61 (1982).

9. M. Jimbo, T. Miwa and M. Okado, Mod. Phys. Lett. B1, 73 (1987).

10. M. Sato, RIMS Kokyuroku 439, 30 (1981).

11. Y. Ohta, R. Hirota, S. Tsujimoto and T. Imai, Jour. Phys. Soc. Japan 62, 1872 (1993).

12. H. Airault, H. McKean and J. Moser, Comm. Pure and Appl. Math. 30, 95 (1977).

13. D. V. Chudnovsky and G. V. Chudnovsky, Nuovo Cimento 40B, 339 (1977).

14. I. M. Krichever, Func. Anal. App. 14, 282 (1980).

15. I. Krichever, O. Babelon, E. Billey and M. Talon, "Spin generalization of the CalogeroMoser system and the Matrix KP equation", preprint LPTHE 94/42.

16. F. Nijhof, O. Ragnisco and V. Kuznetsov, "Integrable time-discretization of the Ruijsenaars-Schneider model", Univ. of Amsterdam preprint 94-27, hep-th/9412170 (1994).

17. I. Krichever, O. Lipan, P. Wiegmann and A. Zabrodin, unpublished.

18. P. P. Kulish, N. Yu. Reshetikhin and E. K. Sklyanin, Lett. Math. Phys. 5, 393 (1981).

19. R. Hirota, J. Phys. Soc. Japan 56, 4285 (1987).

20. Al. B. Zamolodchikov, Phys. Lett. B253, 391 (1991).

21. R. Hirota, J. Phys. Soc. Japan 43, 2079 (1977).

22. L. D. Faddeev and A. Yu. Volkov, Teor. Mat. Fiz. 92, 207 (1992) (in Russian); L. D. Faddeev, "Current-like variables in massive and massless integrable models", Lectures at E. Fermi Summer School, Varenna 1994, hep-th/9406196.

23. A. Bobenko, N. Kutz and V. Pinkall, Phys. Lett. A177, 399 (1993).

24. V. Bazhanov and N. Reshetikhin, J. Phys. A23, 1477 (1990).

25. J.-L. Gervais and A. Neveu, Nucl. Phys. B238, 125 (1984).

26. G. Jorjadze, A. Pogrebkov, M. Polivanov and S. Talalov, J. Phys. A19, 121 (1986).

27. A. Kirillov and N. Reshetikhin, J. Phys. A20, 1565 (1987).

28. V. Bazhanov and N. Reshetikhin, Int. J. Mod. Phys. A4, 115 (1989).

29. R. J. Baxter, Exactly solved models in statistical mechanics (Academic Press, 1982).

30. A. B. Zamolodchikov and Al. B. Zamolodchikov, Ann. Phys. 120, 253 (1979).

31. B. McCoy and T. T. Wu, Phys. Rev. Lett. 45, 675 (1980); Nucl. Phys. B180, 89 (1981).

32. J. H. H. Perk, Phys. Lett. A79, 3 (1980).

33. H. Au-Yang and J. H. H. Perk, Physica 18D, 365 (1986).

34. N. M. Bogoliubov, A. G. Izergin and V. E. Korepin, Quantum inverse scattering method and correlation functions (Cambridge University Press, 1993).

35. V. Bazhanov, S. Lukyanov and A. Zamolodchikov, "Integrable structure of conformal field theory, quantum KdV theory and thermodynamic Bethe ansatz", preprint CLNS 94/1316, RU-94-98, hep-th/9412229. 